\title{
Las redes son dinámicas: explorando cómo las relaciones sociales se entrelazan con la vida
}

\author{
Francisca Ortiz ${ }^{1}$ \\ The Mitchell Centre for Social Network Analysis, The University of Manchester. \\ José Luis Molina ${ }^{1}$ \\ GRAFO- Departamento de Antropología social y cultural, Universitat Autònoma de Barcelona.
}

\section{RESUMEN}

Coincidiendo con la reciente publicación por Cambridge University Press de la versión inglesa del libro de Claire Bidart, Alain Degenne y Michel Grossetti (2020) Living in Networks. The Dynamics of Social Relations presentamos una reseña actualizada de las importantes aportaciones realizadas por los autores tanto para la literatura sobre el ciclo de vida desde una perspectiva relacional como para las perspectivas longitudinales del análisis de redes personales.

Palabras clave: Ciclo de vida - Redes dinámicas - Redes personales - Relaciones.

\section{ABSTRACT}

Coinciding with the recent publication by Cambridge University Press of the English version of the book by Claire Bidart, Alain Degenne, and Michel Grossetti (2020) "Living in Networks. The Dynamics of Social Relations," we present an updated review of the crucial contributions made by the authors both for the literature on the life cycle from a relational perspective and for the longitudinal perspectives of personal network analysis.

Key words: Life Course - dynamic networks - personal networks - relationships.

${ }^{1}$ Contacto con los autores: Francisca Ortiz (francisca.ortiz@manchester.ac.uk / franortizruiz@gmail.com), José Luis Molina (JoseLuis.molina@uab.es) 


\section{INTRODUCCIÓN}

Han pasado 8 años desde la publicación en REDES de la reseña de la versión francesa del libro La vie en réseaux. Dynamique des relations sociales (de Federico 2012). La reciente publicación de la versión inglesa en la colección especializada en análisis de redes sociales de Cambridge University Press representa un gran impulso a su difusión a nivel internacional. Y no es para menos. El libro de Claire Bidart, Alain Degenne y Michel Grossetti (2020) Living in Networks. The Dynamics of Social Relations recoge el saber acumulado sobre las redes personales y sus dinámicas a través del ciclo vital, combinando de forma magistral los fundamentos teóricos y una base empírica realmente excepcional.

Las preguntas planteadas por sus autores son simples y poderosas:

"¿Cómo surgen las redes personales de los contextos sociales? ¿Cómo evolucionan a lo largo de la vida? ¿Cómo se establecen, mantienen, conectan e interrumpen las relaciones? ¿Cómo evoluciona la estructura de una red a medida que las personas afrontan eventos y transiciones?" 1

Para lograr este cometido, los autores utilizan distintas fuentes de datos, en especial dos estudios propios: en primer lugar, la Encuesta de Toulouse $(\mathrm{N}=399)$ basada en el clásico estudio de Claude Fisher (1982) en California. En segundo lugar, el Panel de Caen, que recoge datos longitudinales entre los años 1995 y 2004 con entrevistas cada tres años a sus protagonistas. Además de estas dos fuentes singulares, tienen en cuenta otras secundarias representativas a nivel nacional (Francia). La combinación de testimonios y datos cuantitativos permiten describir e ilustrar de forma convincente las dinámicas relacionales que tienen lugar en el curso vital, así como las consecuencias de las disrupciones, la movilidad o las comunicaciones digitales, entre otros aspectos.

El libro está dividido en tres partes y doce capítulos. En la primera parte, los autores presentan los fundamentos teóricos de una sociología de las dinámicas relacionales. En la segunda parte, con el apoyo de la información empírica, se ilustran los mecanismos que tienen lugar en estas dinámicas relacionales y en su interacción con instituciones y organizaciones. Finalmente, en la tercera

\footnotetext{
${ }^{1}$ Las traducciones entrecomilladas son propias.
}

parte, se analizan las dimensiones del tiempo, el espacio y la digitalización en los procesos relacionales que llevan a la segregación y creación de desigualdades. Veamos cada una de ellas.

\section{LOS FUNDAMENTOS TEÓRICOS}

El primer capítulo se ocupa de estudiar la naturaleza de las relaciones, las redes y los círculos sociales, así como los regímenes de regulación de las interacciones. Las relaciones sociales surgen de contextos de interacción, alcanzan vida propia a nivel diádico, pero también afectan y se ven afectadas por las redes más amplias en las que se insertan.

Siguiendo estos principios, las relaciones sociales se singularizan y desarrollan su propia historia. En este sentido es oportuno recordar la distinción existente entre relación e interacción. En sus palabras:

"La diferencia entre una relación y una interacción es que una interacción es un evento único, mientras que una relación es una serie de interacciones entre los mismos individuos. Si estas interacciones se repiten y se incorporan a determinadas rutinas, pueden dar lugar a familiaridad, expectativas y reconocimiento mutuo." (Bidart, Degenne y Grossetti, 2020: 23)

Así, estas relaciones no son simples nexos entre dos nodos, sino que se desarrollan en el marco de redes y círculos sociales, ayudando a su mantenimiento $y$, eventualmente, también a su disolución.

En el segundo capítulo los autores estudian las redes personales a través de su estructura y composición, así como los indicadores que permiten su medición. En este punto es muy relevante su propuesta de distinción de cuatro tipos diferentes de redes personales atendiendo a su estructura: densas, centradas, disociadas y compuestas. En capítulos posteriores muestran cómo estas configuraciones pueden cambiar de un tipo a otro como consecuencia de eventos vitales y transiciones biográficas, pero que tienen una importante inercia o tendencia a la estabilidad, sobre todo en los dos primeros tipos. 


\section{LAS DINÁMICAS DE LAS RELACIONES SOCIALES}

En esta segunda parte se profundiza en los cambios y transiciones que tienen lugar a lo largo del ciclo vital. Así, en el capítulo 3, se explican los orígenes de las relaciones enmarcándolos en los contextos en los que ego interactúa. El acceso a nuevos contextos permite la aparición de nuevas oportunidades, el distanciamiento de otros y la expansión de la red de ego. A continuación, el capítulo 4 se centra en las microdinámicas relacionales, en concreto en los procesos que explican la multiplexidad, la especialización, la individualización, la incrustación y la disociación, es decir, la combinación de diferentes roles en una misma relación, su especialización, la emancipación de una relación de sus contextos y los procesos de inscrustación y disociación de éstos.

Despues de estudiar cómo se crean, mantienen y comportan las relaciones, los autores pasan a examinar en el capítulo 5 las razones de su desaparición. Entre éstas destacan cinco: desaparición de un contexto o un contacto compartidos, la distancia social, la distancia física y los conflictos que surgen del devenir social. A través de este análisis es posible ver cómo los cambios en relaciones particulares tienen consecuencias en la red en su conjunto. En palabras de los autores:

\begin{abstract}
"La dinámica de las redes personales puede ser el resultado de múltiples procesos, algunos de los cuales dependen directamente de las características de las redes o relaciones en cuestión, mientras que otros surgen de los eventos biográficos que implican una renovación o reconfiguración significativa de las relaciones." (Bidart, Degenne y Grossetti, 2020: 134)
\end{abstract}

En el capítulo 6 los autores analizan los cambios en composición y estructura que se producen a lo largo del ciclo vital, mostrando la existencia de grandes cambios, pero también una relativa estabilidad estructural, especialmente en los tipos densos y centralizados, como ya hemos apuntado. Las transiciones biográficas "interfieren con las redes personales" (Bidart, Degenne y Grossetti, 2020: 165 - traducción propia). Las transiciones más importantes en la vida adulta son: pasar de ser estudiantes a trabajadores, mudarse de casa a vivir en pareja y el nacimiento del/de la primer/a hijo/a. A estas transiciones se suman los cambios propiciados por la movilidad geográfica, el desempleo, un cambio en la trayectoria profesional, interrumpir una relación romántica, o declararse homosexual.

\section{LOS MUNDOS SOCIALES DE LAS REDES}

La tercera parte está destinada a explorar los "mundos sociales" y su mutua influencia con las redes personales. Así el capítulo 8 aborda la dimensión espacial de las redes a través de la exploración de la relación existente entre la proximidad geográfica (dónde viven, trabajan o se socializan), con la proximidad relacional (nivel de cercanía o intensidad entre dos actores). El análisis muestra cómo las redes están casi completamente localizadas en un rango geográfico y cómo los vínculos fuertes ayudan a resistir mejor la separación causada por las grandes distancias. Igualmente, se estudian las diferencias entre las redes urbanas y las rurales, destacando la mayor homifilia en las primeras como consecuencia de la mayor libertad de ego de escoger sus relaciones. Por último, los autores destacan los procesos de reconstrucción social y geográfica de las redes personales motivados por los cambios de residencia, procesos que en pocos años permiten la incorporación de proporciones apreciables de contactos locales.

En el capítulo 9 los autores intentan responder a una de las cuestiones clásicas del análisis de redes: ¿Hasta qué punto las similitudes 0 diferencias estructurales explican la creación y el mantenimiento de las relaciones sociales? Algunos estudios han respaldado la idea de que estas afinidades (género, edad, ocupación, actividades recreacionales, etc.) constituyen los mecanismos de construcción de una sociedad que tiende a una segregación por capas homogéneas. Otros autores, en cambio, defienden que estas afinidades contribuyen a diluir los mundos sociales basados en categorías sociales, comunidades de origen, u otras.

A continuación, en el capítulo 10, los autores revisan la vinculación entre las relaciones sociales y los recursos. En este sentido, las redes han sido tradicionalmente interpretadas en la literatura en términos de los recursos que están disponibles para ego o sus alteri. Esto puede implicar reciprocidad en la ayuda material o el uso instrumental por parte de ego de sus relaciones. Pero también es importante analizar hasta qué punto las decisiones o la conducta de ego pueden estar influenciadas por alteri a los que toma como modelos o con los que discute los temas importantes que le afectan.

Revisadas las fuentes de influencia y agencia en las redes personales, el capítulo 11 se centra en las desigualdades. El lector al Ilegar hasta aquí en el libro ya dispone de un marco teórico y empírico lo suficientemente completo como para que sea necesario volver ahora a un nivel 
más transversal y/o estructural a las redes. Así, los autores presentan cómo varía el número de relaciones, la composición de redes y sus estructuras.

Por último, el capítulo 12 aborda el tema clave de la influencia del contínuo online/offline en las relaciones sociales en general y las redes personales en particular: nuevos contextos de interacción, relaciones débiles y efímeras, reforzamiento de lazos fuertes y el reforzamiento de la homofilia, son algunos de los aspectos aquí considerados.

Una vez finalizada la magna tarea emprendida, los autores presentan ideas, propuestas y sugerencias para futuras investigaciones.

\section{CONCLUSIÓN}

Sin duda alguna, el libro de Bidart, Degenne y Grossetti (2020) es una aportación importante al análisis de redes sociales en general y al estudio de las redes personales en particular. Uno de los aportes de este libro es su perspectiva longitudinal basada en un panel realmente único, combinando datos cuantitativos con testimonios de sus protagonistas. También es un mérito innegable el análisis de las redes desde los propios contextos y los círculos sociales en las que se insertan estas transiciones vitales. Así, leyendo este libro se dispone de una visión informada, rigurosa y completa del mundo de las redes personales y su dinámica a través del ciclo vital. En suma, esta obra se suma a la importante colección de contribuciones al análisis de redes personales con diferentes énfasis y perspectivas, como los escritos por Crossley et al. (2015), Perry, Pescosolido y Borgatti (2018), y McCarty et al. (2019, ver Maya-Jariego, 2021). En relación con estas obras, este libro nos permite entender los mecanismos que de forma dinámica afectan no solamente a las transiciones vitales individuales sino a los círculos sociales y las organizaciones en las que estas trayectorias se insertan y, a su vez, constituyen. Gracias y felicidades.

\section{AGRADECIMIENTOS}

Agencia Nacional en Investigación y Desarrollo de Chile ANID [2018 - 72190281].

\section{REFERENCIAS}

Bidart, C., Degenne, A. and Grossetti, M. (2020). Living in Networks. The Dynamics of Social Relations. Cambridge, United Kingdom: Cambridge Press.

Carrington, P., Scott, J. and Wasserman, S. (2005). Models and methods in Social Network Analysis. Cambridge, United Kingdom: Cambridge Press.

Crossley, N., Bellotti, E., Edwards, G., Everett, M, Koskinen, J. y Tranmer, M. (2015). Social Network Analysis for Ego-Nets. London, United Kingdom: SAGE Publications.

Domínguez, S. and Hollstein, B. (2014). Mixed methods in Social Network Research. Design and applications. Cambridge, United Kingdom: Cambridge Press.

de Federico, A. (2012). Recensión del libro "La vie en réseau. Dynamique des relations sociales" de Claire Bidart, Alain Degenne y Michel Grossetti, REDES-Revista Hispana para el Análisis de Redes Sociales 23(2): 291-296.

Fisher, C. (1982). To Dwell among friends. Personal networks in town and city. Chicago, United States: The University Chicago Press.

Maya-Jariego, I. (2021). Una guía práctica para realizar estudios de redes personales. REDES-Revista Hispana para el Análisis de Redes Sociales, 32(2):219-222.

McCarty, C., Lubber, M., Vacca, R. y Molina, J.L. (2019). Conducting personal networks research. A practical guide. New York, United States: The Guildford Press.

Perry, B., Pescosolido, B. y Borgatti, S. (2018). Egocentric network analysis. Foundations, methods and models. Cambridge, United Kingdom: Cambridge Press.

Wasserman, S. and Faust, K. (1995). Social Network Analysis. Methods and applications. Cambridge, United Kingdom: Cambridge Press. 